\title{
Database visualization
}

\author{
MARC ONDRECHEN \\ Silicon Graphics Computer Systems, Minneapolis, Minnesota
}

\begin{abstract}
A typical database contains a wealth of data of which a user is unaware or unable to access. Converting these data into useful, meaningful information has previously been extremely difficult and haphazard. The availability of visualization software coupled with high-performance, multiprocessing Challenge data servers from Silicon Graphics, Inc., has enabled users to interact with their databases in real time, dramatically increasing the amount of information they can extract from their databases.
\end{abstract}

\section{The Evolution of Silicon Graphics Computer Systems}

Silicon Graphics, Inc. (SGI) offers a wide range of computer products, which include general-purpose desktop computers, computer-aided design (CAD) workstations, graphics supercomputers, file and computational servers, and arrays of multiprocessor compute servers. All of these computers use a common operating system which makes it easier for users to administer and interact with any of our computer systems. SGI was founded in 1982 to create graphics workstations, and to allow users to "see" their data, instead of just looking at numbers on a piece of paper. This concept was termed visual computing, and today it has become commonplace. SGI's success with graphics has had an unintended side effect--people often are unaware that SGI has become a general-purpose computer company. Recently, SGI has received a large amount of press coverage due to the role played by SGI computers in the movie industry and by the special effects enabled by the computer technology pioneered and manufactured by SGI. This has changed SGI's reputation somewhat, and focused attention on the entertainment markets. However, SGI's revenues still come predominantly (about $70 \%$ ) from engineering, visual simulation, and scientific visualization applications. SGI's merger with Cray Research has created the world leader in technical computing, and SGI is clearly no longer just a graphics company!

The demands of providing real-time, interactive threedimensional computer graphics has led to the development of computer systems able to excel in a wide range of often unexpected applications. SGI was the first workstation vendor to adopt reduced instruction set computing (RISC) technology in response to the demands of graphics processing. This decision led to the creation of new workstations, capable of computational tasks previously left to mainframe computers, and to large file servers. As the needs of visual computing increased, demands on all aspects of the computer system increased. Faster disk access, larger files, and increased input/output ( $\mathrm{I} / \mathrm{O})$ capac-

M. Ondrechen's address is Silicon Graphics Computer Systems, 5601 Green Valley Dr., Bloomington, MN 55437 (e-mail: marc@minneapolis. sgi.com). ity were required. And, as it turned out, the systems produced to satisfy these demands would serve the needs of other applications as well. These systems are especially well suited to the needs of databases, where faster I/O, multiple central processing units (CPUs), and large data sizes allow users to query and receive answers in real time rather than in minutes or hours.

Once the power of SGI's Challenge servers was recognized by database software vendors, they quickly became those vendors' computers of choice for showing off their software (e.g., Oracle and Sybase). It also became clear that combining the compute power with the graphics power of SGI systems would lead to another revolution in computing - the advent of real-time interactive visualization of databases and datasets.

\section{Database Visualization}

As an example of the usefulness of database visualization, let us look at some applications. Imagine a grocery store that wants to know what items are most commonly purchased and what correlations there might be between those items and possibly any other items in the store. Cash-register receipts can of course be saved and analyzed, but reading through numerical output is a timeconsuming task. Viewing such data graphically, colorcoded for frequency of purchase, allows a store manager to instantly recognize and identify items that are most commonly purchased.

In 1989, engineers at AT\&T were interested in verifying that the switching software used for handling longdistance calls behaved as it was designed to. They were particularly interested in knowing how the software would respond to a situation where calls going to a particular location exceeded the capacity of the destination's local phone system. This is impossible to effectively simulate under normal conditions. Fortunately for the AT\&T engineers (though unfortunately for millions of other people), the Bay Area earthquake in 1989 provided the perfect testing environment. By using a graphical interface to view the massive amount of call data, the engineers could easily identify call overloads and switch responses to those overloads. Furthermore, they could interactively watch how the switches worked over time, stopping the 
replay of data at any point in time to view a specific switch, and so on. The switches worked nearly flawlessly, and this made the engineers quite happy.

But the rest of the story involves AT\&T's marketing department, and provides an illustration of the fact that information contained in databases is often unknown to or unrecognized by the user. As it turned out, a large number of calls to the Bay Area originated in the Seattle area and in Florida. This gave the marketing people key demographic information - if they wanted to target people who might call the Bay Area, Seattle and Florida were good places in which to advertise. A possible slogan for the campaign might be "Call your loved ones in the Bay Area before the next Big One hits" (see Hadary, 1995). A bit of dark humor, perhaps, but the point that the engineers' data contained data that were useful to Marketing as well is significant.

Another useful example from AT\&T's history with long-distance phone service involves illegal routing from one country to another. In this instance, when people in one country wished to call another country (but were officially prohibited by their local government from doing so) and called a number in the United States, they were routed to their final destination. AT\&T was not concerned that those citizens were violating their country's laws, but they did object to the fact that AT\&T was not being paid for the routed calls! In the past, these illegal routes were detected only after the fact, when analyzing the previous month's phone bills. By using the real-time database visualizer, they could watch call patterns develop during the month, and when the characteristic call pattern appeared, could quickly terminate service at that location. These call patterns look like spaghetti to most people, but are quite obvious to AT\&T engineers. Early disconnection saves AT\&T thousands of dollars each time the patterns are detected.

A final example involves both AT\&T and $\mathrm{MCI}$ in the battle for long-distance service. Both companies are constantly claiming lower rates, better service, and so on. In a novel marketing strategy, AT\&T took advantage of the power of SGI's Challenge servers by connecting them to the database containing the previous month's long-distance calls made by all customers in the United States. They then called competitors' customers and compared rates while on the phone with them. Surprisingly, AT\&T rates were often better! In the first month, AT\&T had recovered thousands of customers, and they were so successful that MCI ordered an identical system from SGI in order to do the same thing to AT\&T's customers.

It is no coincidence that most of the cited examples above involve phone companies. Long-distance service is extremely competitive, and even a slight edge in technology or services can translate into annual savings of millions of dollars. The Challenge line of servers provides that competitive edge, not only to MCI and AT\&T, but to anyone who needs rapid access to, and comprehension of, massive amounts of stored data. The ability to view these data graphically and in real time is only now being realized and effectively exploited.

\section{Graphical User Interfaces for Databases}

A number of graphical user interfaces to databases are available or being developed. SGI's MineSet is one, as is Visual Information Discovery from AT\&T Capital Corporation. Generally, these products are in the form of modules and groups of libraries and subroutines. In this way, the desired interface can be readily created from a set of standard tools. This approach is desirable, since most database users would prefer a customized graphical user interface. In the examples cited above, each data set was different, as were the questions being asked. Thus, it was desirable to be able to create interfaces that visually displayed the data differently but otherwise maintained common elements in the user interface. This makes it easy for users to work with any of the data sets.

\section{Summary}

Graphical user interfaces to databases are becoming increasingly popular, and a number of tool sets are now available to users of most commercial databases. These tool sets allow users to quickly develop customized visualization front ends to their databases. With the processing power of SGI servers, accessing and querying a database becomes an interactive, iterative process, with quicker and easier-to-comprehend responses.

\section{REFERENCE}

HADARY, J. (1995). Database visualization white paper. AT\&T Capital Corporation. (Available: Insight Solutions, Inc., 6310 Highland Place, Sebastapol, CA 95472) 